\title{
Hydrodissection of an achilles tendinopathy on a professional athlete
}

\begin{abstract}
We report a case of Achilles tendinosis that was treated with a high volume, hydrodissection visualized by conventional B-mode ultrasound. Prior MRI revealed a thickened right Achilles tendon with a small defect. Ultrasound examination showed a thickened Achilles tendon with physical exam revealing a prominent Haglund's deformity, mild fusiform swelling and pain along the posterior Achilles (Figure $1 \& 2$ ). The condition had been poorly responsive to standard management including protected ambulation in a cam walker boot, anti-inflammatory medication, modalities and therapeutic exercise, as well as a platelet rich plasma injection. We suggest using high volume ultrasound guided injection adjacent to (anterior to) the Achilles tendon as an adjunct to treat this difficult clinical syndrome.
\end{abstract}

Keywords: Hydrodissection; Achilles tendinitis; High-volume injection; Haglund's deformity; Tendinopathy
Volume 8 Issue I - 2017

\author{
Deryk Jones, P Gunnar Brolinson, Scottie \\ Patton, Christina Wong \\ New Orleans Saints National Football League, Ochsner Clinic, \\ Edward Via College of Osteopathic Medicine and Virginia Tech \\ Sports Medicine, USA
Correspondence: Deryk Jones, New Orleans Saints Nationa Football League, Ochsner Clinic, Edward Via College of Osteopathic Medicine and Virginia Tech Sports Medicine, USA, Email djones@ochsner.org

Received: October 26, 2016 | Published: April 19, 2017

\section{Introduction}

Achilles tendinitis is a common acute and chronic injury amongst both athletes and non-athletes. Up to $45 \%$ of patients do not respond to standard medical management. ${ }^{1,2}$ Treatment for this injury includes many different options including pharmacotherapy, injections, shoe lifts, physical therapy, electrotherapy, and surgery as a last resort. ${ }^{3}$ D.P. is a 23 y.o. African American male professional football player with intermittent right Achilles tendon pain for one year. He first began to have discomfort during his senior season of college football which partially responded to standard management and relative rest. He did not miss any games, but had modified workouts in practice and training. Toward the end of his first mini-camp he had a flare up but felt that it would resolve with rest. A MRI was obtained showing mild fusiform swelling of the distal Achilles tendon and a small defect. He remained symptomatic and ultimately had a PRP injection. During the first week of preseason camp, he had another flare up and was seen in consultation by a foot and ankle specialist who suggested protected ambulation with a cam walker boot, rehabilitative exercises, and restriction from competitive football activities for 8-12 weeks. In further discussion, the athlete requested a more aggressive conservative management approach as he wished to return to competitive football activities as soon as possible. His past medical history was remarkable only for a previous right knee arthroscopic partial lateral meniscectomy in 2014. Lower extremity alignment revealed moderate bilateral pes planus with valgus calcaneal positioning with associated mild valgus knee alignment. We discussed hydrodissection with him as an adjunct to management and this case report considers high volume injection with D5 normal saline mixed with $1 \%$ lidocaine without steroid into the interface of the retrocalcaneal bursa and tendon along with low volume injections of D5 normal saline along the course of the sural nerve as a form of adjunctive management for recalcitrant Achilles tendinitis and tendinopathy.

\section{Materials and methods}

To perform the hydrodissection of the Achilles' tendon, the tendon was first visualized under B-mode ultrasound by an experienced user with a linear probe. The area was prepped with betadine and sterile ultrasound gel, and the probe was covered to keep it sterile. The length of the fusiform swelling seen on ultrasound and length of palpable tenderness on physical was marked with a skin pen. Additionally, the sural nerve was visualized and the path was marked every $1 \mathrm{~cm}$ along on the skin laterally extending $1 \mathrm{~cm}$ above and below the maximum tenderness. Using a 21 gauge, $1 \frac{1 / 2}{2}$ " needle, two syringes the of $10 \mathrm{cc}$ D5 normal saline and $1 \mathrm{cc}$ of $1 \%$ lidocaine without epinephrine were injected on the lateral aspect of the posterior ankle under direct ultrasound guidance to separate the anterior Achilles tendon from Kager's fat pad. Two points of entry were made to separate these layers, along the length of the swelling and tenderness of the tenderness. Next, using a 27 gauge, $1 / 2$ " needle, $0.5 \mathrm{cc}$ of D5 normal saline was injected into each four points along the marked path of the sural nerve.

\section{Results}

The player in this case study reported immediate improvement of his pain from an " 8 " down to a " 2 ", he was able to ambulate without discomfort and did not have any tenderness to palpation at the Haglund's deformity after the procedure. He continued to wear his cam walker boot and maintained his rehabilitation plan with his athletic trainer starting the next day. He did report gradual increase in his pain (though decreased from before the hydrodissection) over the following week but was able to participate in a more aggressive rehabilitative program. He underwent a second hydrodissection one week later and again reported and even lower pain scores (from a " 6 " down to a "1").

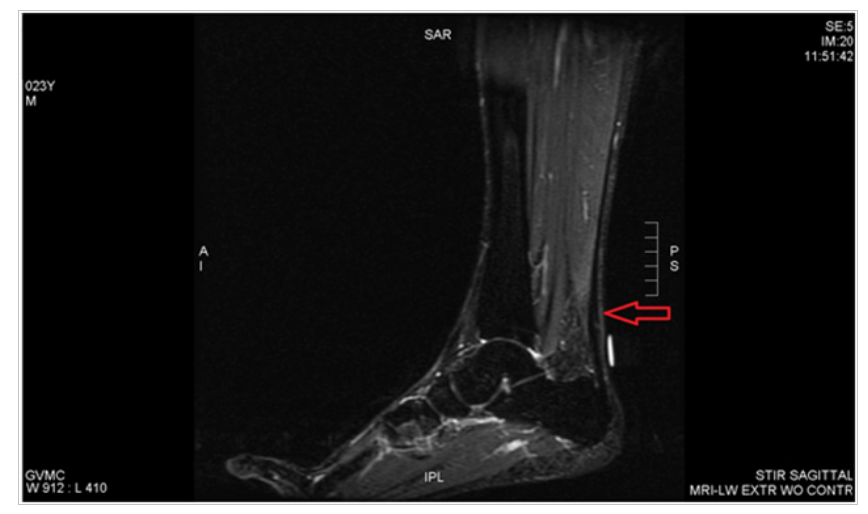

Figure I T2, sagittal imaging shows prominent Haglund's deformity and fusiform swelling. 


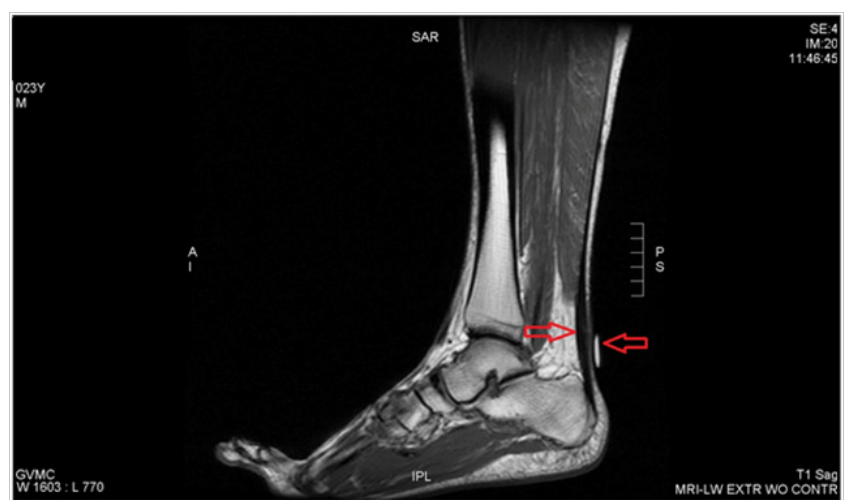

Figure 2 TI, sagittal imaging shows prominent Haglund's deformity and fusiform swelling.

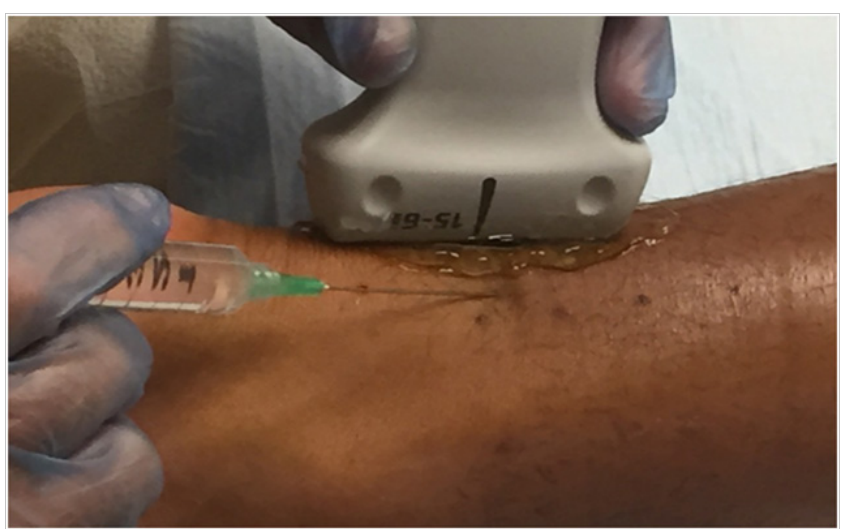

Figure 3 Lateral view of the hydrodissection of the Achilles tendon with the sural nerve clearly marked.

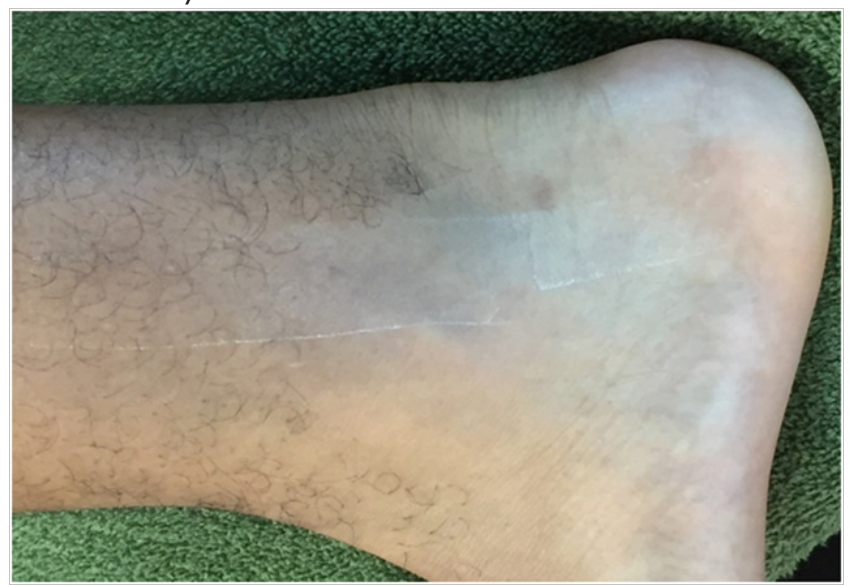

Figure 4 Haglund's deformity on physical exam.

\section{Discussion}

Achilles tendinosis a common cause of posterior ankle pain. The blood flow at the calcaneal insertion is considerably lower than the rest of the Achilles tendon when visualized by Doppler. Tendinosis is the preferred terminology, rather than tendinitis, as tendinosis refers to a disruption of the collagen tissue rather an actual inflammatory process. Many factors play into who may develop tendinosis, including age, sex, mechanical overload and overuse activities, poor flexibility, and other endocrine problems. ${ }^{4}$

Conservative treatment is recommended as many patient's pain and function will improve. Frequently, however, this condition will be poorly responsive to standard management resulting in chronic and recurring symptoms which limit a patient's ability to participate in competitive athletics. As shown in the case above, performing a hydrodissection of the Achilles can be used in adjunct to other treatments such as physical therapy, non-steroidal anti-inflammatories, and platelet-rich plasma (PRP) injections prior to considering surgical intervention. ${ }^{3}$ Potentially, a hydrodissection may help an athlete tolerate a more aggressive physical therapy or rehabilitation program in order to maintain strength, flexibility and range of the motion in the affected lower extremity (particularly stretching exercises).

One theory behind why hydrodissection may improve pain is that it separates scar tissue formed between the Achilles and Kager's fat pad. This scar tissue may cause pain in the posterior ankle and restrict the flexibility of the tendon during movement and exercise. The influx of solution between the fat pad and Achilles tendon removes the tethering factors that prevent the natural fascial glides between the different layers of tissue. ${ }^{3}$

In February 2016, a case series of three patients received plateletrich plasma, prolotherapy, anesthetic and steroid injected in or around the Achilles tendon. ${ }^{5}$ All three patients received hydrodissection during each injection procedure. Two of the three were symptom free immediately after the procedure and the third was pain free the day after. The case review was limited due to a small sample size; however, all three methods (regardless of solution/substance used) performed hydrodissection and showed symptomatic improvement. A second study comparing lower volume $(30 \mathrm{~mL}$ saline $/ 1 \%$ lidocaine mixture) to higher volume $(50 \mathrm{~mL}$ saline $/ 1 \%$ lidocaine mixture) found that higher volumes injected into the ankle to perform a hydrodissection of Kager's fat pad and Achilles tendon had improvement in both pain scores and overall function, based on a 100 point scale called the Victorian Institute of Sports Assessment - Achilles (VISA-A) questionnaire. ${ }^{6}$

It is possible that any volume hydrodissection may even be of benefit over PRP, given that a third study comparing PRP to placebo (saline) injections into the Achilles tendon did not show any difference in VISA-A score over 3 months. ${ }^{7}$ These results were limited due to large patient drop-outs after the first 3 months, with a goal of 12 month study. Although saline injections were used as the placebo, they were relatively low volumes $(6 \mathrm{~mL}$ of $0.9 \%$ normal saline as opposed to $22 \mathrm{~mL}$ of D5 normal saline used in this case report) and saline injections were used to perforate through the tendon as opposed to separating the Achilles and Kager's fat pad.

\section{Conclusion}

Hydrodissection of the Achilles tendon can be considered as an adjunctive treatment for acute or chronic Achilles tendinosis. Future studies and case reports should continue to evaluate the usefulness of this novel treatment in the management and return to play of athletes with this difficult to treat condition.

\section{Acknowledgments}

New Orleans Saints and Academic Sports and Osteopathic Medicine.

\section{Conflicts of Interset}

None.

\section{References}

1. Al fredson H, Pietlia T, Jonsson P, et al. Heavy-Load Eccentric Calf Muscle Training For the Treatment of Chronic Achilles Tendinosis. The American Journal of Sports Medicine. 1998;26(3):360-366. 
2. Malliaras P, Barton C, Reeves N, et al. Achilles and Patellar Tendinopathy Loading Programmes: A Systematic Review Comparing Clinical Outcomes and Identifying Potential Mechanisms for Effectiveness. Sports Med. 2013;43(4):267-286.

3. Alfredson H, Cook J. A treatment algorithm for managing Achilles tendinopathy: new treatment options. British Journal Sports Medicine. 2007;41(4):211-216.

4. Kader D, Saxena A, Movin T, et al. Achilles tendinopathy: some aspects of basic science and clinical management. British Journal Sports Medicine. 2002;36(4):239-249.
5. He L, GeninJ, Delzell P. Ultrasound diagnosis and percutaneous treatment of Achilles tendon tethering: a case series. Skeletal Radiol. 2016;45(9):1293-1298.

6. Wheeler P, Mahadevan D, Bhatt R, et al. A Comparison of Two Different High-Volume Image-Guided Injection Procedures for Patients with Chronic Noninsertional Achilles Tendionpathy: A Pragmatic Retrospective Cohort Study. J Foot Ankle Surg. 2016;55(5):976-979.

7. Krogh T, Ellingsen T, Christensen R, et al. Ultrasound-Guided Injections Therapy of Achilles Tendinopathy with Platelet-Rich Plasma or Saline: A Randomized, Blinded, Placebo-Controlled Trial. The American Journal of Sports Medicine. 2016;44(8):1990-1997. 